\title{
A nano-fiber coupler thermometer
}

\author{
W. Talataisong*, R. Ismaeel, M. Beresna and G. Brambilla \\ Optoelectronics Research Centre, University of Southampton, Southampton, SO17 1BJ, UK \\ *Corresponding author: w.talataisong@soton.ac.uk
}

\begin{abstract}
Optical fiber couplers with $\sim 300 \mathrm{~nm}$ waist diameter are used as compact temperature sensors from room temperature to $661^{\circ} \mathrm{C}$, showing an average temperature sensitivity of $55 \mathrm{pm} /{ }^{\circ} \mathrm{C}$ and a maximum sensitivity of $60 \mathrm{pm} /{ }^{\circ} \mathrm{C}$ at the highest temperature.
\end{abstract}

Keywords-nanofiber coupler; temperature sensor; broad temperature; large dynamic range

\section{INTRODUCTION}

Optical fiber temperature sensors have been investigated for many applications including oil and gas industry, electric circuits health monitoring and structural health monitoring. Fiber Bragg gratings (FBG) and long period gratings (LPG) are the most commonly used optical fiber point temperature sensors $[1,2]$. However, FBG and LPG require specialty fibers, a long manufacturing process, and exhibit a relatively slow response time. Recently optical microfibers have been investigated for temperature sensing because of their compactness, quick manufacturing, fast response time and high sensitivity [3-5] Here we demonstrate a nanofiber coupler (NCF) as a high sensitivity temperature sensor. The NCF operates in two mode beating regime allowing to maintain high sensitivity over large range of temperatures.

\section{PRINCIPLE}

The typical fiber coupler is composed of two input ports $\left(\mathrm{P}_{1}\right.$ and $\left.\mathrm{P}_{2}\right)$ and two output ports $\left(\mathrm{P}_{3}\right.$ and $\left.\mathrm{P}_{4}\right)$ (Fig. 1).

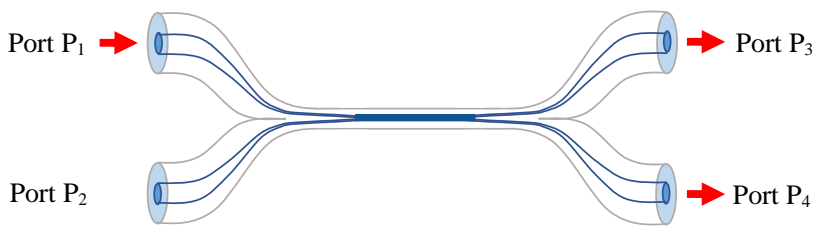

Figure 1. Schematic of a bi-conical fiber coupler.

In the coupling region, the beating of supermodes with different propagation constants provide a continuous change in the power distribution along the coupler cross section, which results in a different power splitting at the output ports for different optical paths. In 2009, a fiber coupler (MFC) with waist diameter in the region of few microns was fabricated and used as a broadband modal filter [6]. Although the coupler supported multiple supermodes, only the first two were excited, resulting in the singlemode operation at its fiber pigtails. When the diameter (d) of the optical fiber coupler is smaller than 1 micron, only the first and second supermodes exist in the coupler and at $\mathrm{d} \sim 300 \mathrm{~nm}$ only one supermode is supported in the third telecom window (Fig. 2). In the multimode region at $\lambda<1520 \mathrm{~nm}$, the beating between two supermodes gives oscillations with high extinction ratios. In the cut-off region $(1520 \mathrm{~nm}<\lambda<1560 \mathrm{~nm})$, the two supermodes stop beating as one of the supermodes is weakly guided because of the small diameter of the coupler. This results in a bump with flat transmission over nearly $40 \mathrm{~nm}$. At longer wavelengths, only one supermode is supported by the NCF, resulting in a constant output at both ports, as shown in the single-mode region in figure 2 .

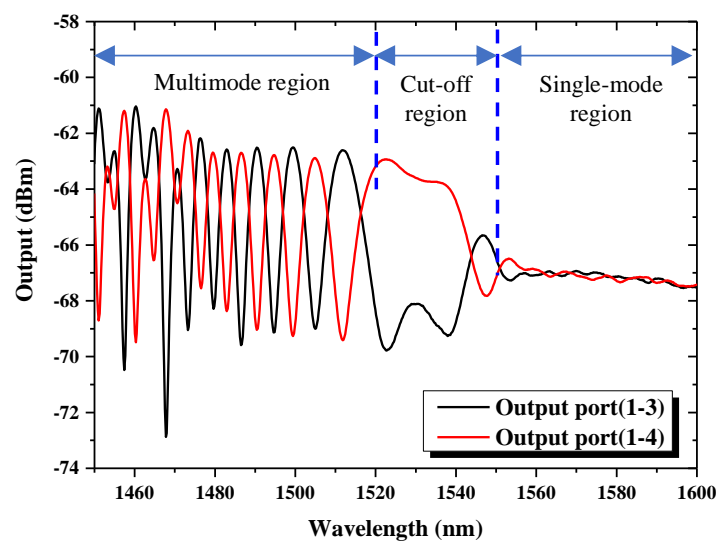

Figure 2. Ouput spectrum of the optical nanofiber coupler with a waist diameter of $300 \mathrm{~nm}$.

The modes optical paths depend both on the coupling region length and its refractive index; since both depend on the surrounding environment refractive index thus on its temperature, the NFC can be used as a highly sensitive temperature sensor.

\section{EXPERIMENTAL SETUP}

The nanofiber coupler (NFC) was fabricated from two telecom fibers (Corning SMF-28) using the modified flame brushing technique [7]. The two single mode fibers were stripped of their acrylic coating over a length of $\sim 50 \mathrm{~mm}$ and twisted 1.5 turns around each other. The twisted region was fused together using a resistive ceramic micro-heater with an estimated temperature of $\sim 1450^{\circ} \mathrm{C}$. In the experiment, the length of the uniform waist region was $4 \mathrm{~mm}$ with a waist diameter of $\sim 0.3 \mu \mathrm{m}$.

In the temperature measurement, the same microheater used to fabricate the NFC was used to apply heat to the sensor. The coupling region of the NFC was inserted into the center of the microheater. Light from an incoherent white light source (Bentham, WLS100) was launched into port 1 of the NFC and the output of port 3 was connected to an optical spectrum

W.Talataisong gratefully acknowledges the Development and Promotion of Science and Technology Talent Project (DPST) of Thailand for her financial support. 
analyzer (Yokogawa, AQ6370) to monitor in real time the transmission spectrum. The microheater temperature was changed by increasing the current from $0.2 \mathrm{~A}$ to $1.2 \mathrm{~A}$ in steps of $0.1 \mathrm{~A}$. The increase of the applied current caused an increase of the microheater temperature from $\mathrm{T}=84^{\circ} \mathrm{C}$ to $\mathrm{T}=661^{\circ} \mathrm{C}$. In the experiment, the current was maintained for 15 minutes at each step to ensure that the microheater temperature is stabilized.

\section{EXPERIMENTAL RESULTS AND DISCUSSION}

To evaluate the sensitivity of the nanofiber coupler thermometer, the three transmission wavelength dips which are close to the cut-off region at the lowest temperature $\left(84^{\circ} \mathrm{C}\right)$ were monitored (Fig. 3). The wavelengths of dip1, dip2, and dip3 are $1505 \mathrm{~nm}, 1495 \mathrm{~nm}$, and $1487 \mathrm{~nm}$, respectively. A red shift of transmission spectrum can be clearly observed for increasing temperatures. At the temperature of $\mathrm{T}=478^{\circ} \mathrm{C}$ dip 1 moved into the cut-off region and the weak guidance of the odd supermode resulted in the disappearance of dip1. Similarly, dip2 disappeared at the $\mathrm{T}=661^{\circ} \mathrm{C}$.

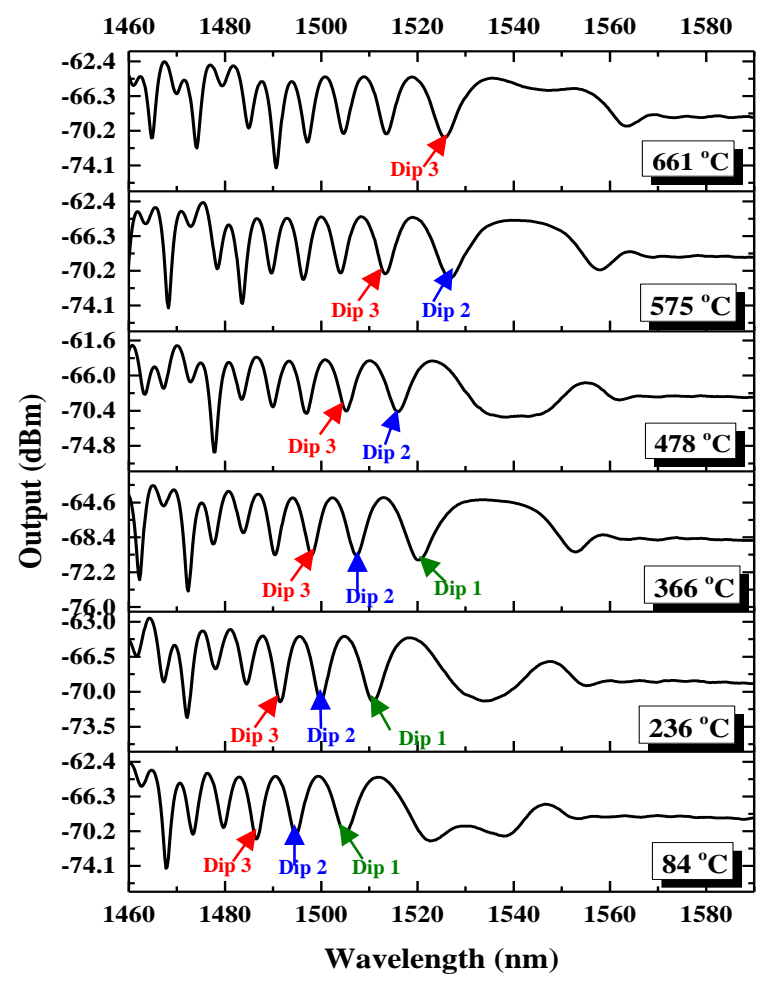

Figure 3. Optical nanofiber coupler transmission spectrum at different applied temperatures.

Results indicate that the wavelength of dip1 can be used to measure temperatures from 0 to $424^{\circ} \mathrm{C}$, while those of dip 2 and dip3 up to the temperatures of $620^{\circ} \mathrm{C}$ and $661^{\circ} \mathrm{C}$, respectively (Fig. 4). By fitting a polynomial function of second degree, the slope of dip1 at the highest temperature of $424^{\circ} \mathrm{C}$ was calculated to be equal $50.80 \mathrm{pm} /{ }^{\circ} \mathrm{C}$ while the slope of $\operatorname{dip} 2$ and $\operatorname{dip} 3$ at the highest temperatures of each dip were calculated to be equal $60.17 \mathrm{pm} /{ }^{\circ} \mathrm{C}$, and $55.23 \mathrm{pm} /{ }^{\circ} \mathrm{C}$, respectively.
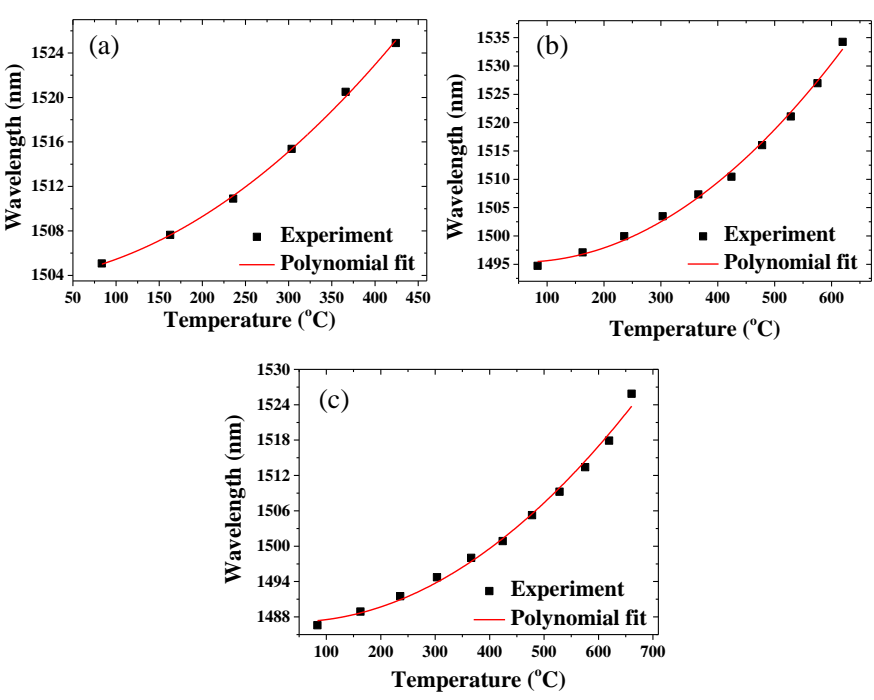

Figure 4. Relationship between wavelength and applied temperature for different wavelength dips (a) dip1 (b) dip 2 (c) dip 3.

To compare the temperature sensitivity of three wavelength dips, the slope of each graph was measured at the same temperature of $424{ }^{\circ} \mathrm{C}$. In the temperature range of $0-424{ }^{\circ} \mathrm{C}$, the sensitivity of $\operatorname{dip} 1, \operatorname{dip} 2$, and $\operatorname{dip} 3$, are $50.80 \mathrm{pm} /{ }^{\circ} \mathrm{C}, 37.21$ $\mathrm{pm} /{ }^{\circ} \mathrm{C}$, and $33.14 \mathrm{pm} /{ }^{\circ} \mathrm{C}$, respectively.

The result shows that spectral features at longer wavelengths, especially those in close proximity to the cut-off region, exhibit a higher temperature sensitivity than those at shorter wavelengths. Yet, wavelength dips closer to the cut-off region present a smaller dynamic range.

In conclusion, By monitoring the wavelength of dip3 with the increasing of the temperature, a thermometer based on the NFC with the large dynamic range and a hight temperature sensitivity of $55.23 \mathrm{pm} /{ }^{\circ} \mathrm{C}$ has been achieved. This is significant improvement compared with earlier demonstrations with a micron-size fibre coupler thermometers [3].

\section{REFERENCES}

[1] Chamorro Enríquez, D.A., da Cruz, A.R., and Rocco Giraldi, M.T.M.: 'Hybrid FBG-LPG sensor for surrounding refractive index and temperature simultaneous discrimination', Optics \& Laser Technology, 2012, 44, (4), pp. 981-986

[2] Li, Y., Yang, M., Wang, D.N., Lu, J., Sun, T., and Grattan, K.T.V.: 'Fiber Bragg gratings with enhanced thermal stability by residual stress relaxation', Opt. Express, 2009, 17, (22), pp. 19785-19790

[3] Ding, M., Wang, P., and Brambilla, G.: 'A microfiber coupler tip thermometer', Opt. Express, 2012, 20, (5), pp. 5402-5408

[4] Guo, H., Pang, F., Zeng, X., Chen, N., Chen, Z., and Wang, T.: 'Temperature sensor using an optical fiber coupler with a thin film', Appl. Opt., 2008, 47, (19), pp. 3530-3534

[5] Wang, P., Ding, M., Bo, L., Guan, C., Semenova, Y., Wu, Q., Farrell, G., and Brambilla, G.: 'Fiber-tip high-temperature sensor based on multimode interference', Opt. Lett., 2013, 38, (22), pp. 4617-4620

[6] Jung, Y., Brambilla, G., and Richardson, D.J.: 'Optical microfiber coupler for broadband single-mode operation', Opt. Express, 2009, 17, (7), pp. 5273-5278

[7] Brambilla, G.: 'Optical fiber nanowires and microwires: a review', Journal of Optics, 2010, 12, (4), pp. 043001 